\title{
ULTRA-IRREDUCIBILITY OF INDUCED REPRESENTATIONS OF SEMIDIRECT PRODUCTS
}

\author{
HENRIK STETKÆR
}

\begin{abstract}
Let the Lie group $G$ be a semidirect product, $G=S K$, of a connected, closed, normal subgroup $S$ and a closed subgroup $K$. Let $\Lambda$ be a nonunitary character of $S$, and let $K_{\Lambda}$ be its stability subgroup in $K$. Let $I^{\Lambda \mu}$, for any irreducible representation $\mu$ of $K_{\Lambda}$, denote the representation $I^{\Lambda \mu}$ of $G$ induced by the representation $\Lambda \mu$ of $S K_{\Lambda}$. The representation spaces are subspaces of the distributions.

We show that $I^{\Lambda \mu}$ is ultra-irreducible when the corresponding Poisson transform is injective, and find a sufficient condition for this injectivity.
\end{abstract}

\section{INTRODUCTION}

Let $G$ be a Lie group which is a semidirect product, $G=S K$, of a connected, closed, normal subgroup $S$ and a compact subgroup $K$. Let $\Lambda$ be a continuous homomorphism of $S$ into $\mathbb{C} \backslash\{0\}$, and let $M:=K_{\Lambda}$ be its stability subgroup in $K$. For any continuous irreducible representation $\mu$ of $M$ on a complex vector space $H(\mu)$ we shall consider the representation $I^{\Lambda \mu}$ of $G$ induced by the representation $\Lambda \mu$ of $S M . I^{\Lambda \mu}$ is a (not necessarily unitary) representation of $G$ on a subspace of the Hilbert space $L^{2}(K, H(\mu))$, viz. on the subspace $L_{\mu}^{2}(K, H(\mu))$ consisting of the vectors $f$ which satisfy the convariance condition

$$
f(m k)=\mu(m)[f(k)] \text { for } m \in M \text { and } k \in K .
$$

The classical unitary theory, which will not be discussed here, is primarily due to Mackey. The special case of the Cartan motion group is treated in detail in [Gi]. In the nonunitary case several authors have studied the question of irreducibility of $I^{\Lambda \mu}:[\mathrm{Th}, \mathrm{Wi}, \mathrm{Ra}$ ] are concerned with topological irreducibility for general semidirect products, and [CD] with topologically complete irreducibility in the case of the Cartan motion group. Nonunitary representations of the Car$\tan$ motion group on eigenspaces of invariant differential operators are studied in [He].

Received by the editors September 1, 1988. Presented on June 6,1989 at the Conference on Harmonic Analysis, Tuczno, Poland, organised by Wroclaw University, Poland.

1980 Mathematics Subject Classification (1985 Revision). Primary 22E45; Secondary 22D30.

Key words and phrases. Lie group, semidirect product, nonunitary representation, induced representation, ultra-irreducibility, Poisson transform, distribution space. 
The present paper deals with the stronger notion of ultra-irreducibility of $I^{\Lambda \mu}$ for general semidirect products with applications to the Cartan motion group, and the subgroup $K$ need no longer be compact.

Our point of view is that there is no need for the Hilbert space frame once we accept nonunitary representations. Any $I^{\Lambda \mu}$-invariant space $E$ of functions or distributions on $K$ satisfying $(*)$ should be studied; the space of square integrable functions satisfying $(*)$ is just a special case. More precisely we let $E$ be an $I^{\Lambda \mu}$-invariant subspace of the distributions $\mathscr{D}^{\prime}(K, H(\mu))$ such that

$$
C_{\mu}^{\infty}(K, H(\mu)) \subset E \subset \mathscr{D}_{\mu}^{\prime}(K, H(\mu)),
$$

where the subscript $\mu$ indicates that the elements should satisfy $(*)$. Particular cases are the extreme ones $C_{\mu}^{\infty}(K, H(\mu)), \mathscr{D}_{\mu}^{\prime}(K, H(\mu))$ and

$$
E=C_{\mu}(K, H(\mu)), L_{\mu}^{p}(K, H(\mu)) \text { for } K \text { compact and } 1 \leq p<\infty .
$$

If $I^{\Lambda \mu}$ is ultra-irreducible on one of the $I^{\Lambda \mu}$-invariant spaces between $C_{\mu}^{\infty}(K, H(\mu))$ and $\mathscr{D}_{\mu}^{\prime}(K, H(\mu))$, it is so on all the others (Theorem II.6).

We define a Poisson transform for a semidirect product as above, and find a sufficient, algebraic condition on it to be injective (Theorem IV.2). The condition is weaker than certain of the conditions found in the literature (e.g., [Th, Theorem 4; Wi, Theorem 5.3; Ra, §2.4]) and holds for the Cartan motion group.

By help of Litvinov and Lomonosov's version of Burnside's theorem [LL1] we establish that, when the Poisson transform is injective, then the representation $I^{\Lambda \mu}$ is ultra-irreducible (Theorem V.2).

Finally we get as a corollary of the above results that those representations of the Cartan motion group which are studied by Champetier and Delorme in [CD], are ultra-irreducible (Theorem VI.1).

\section{GROUP REPRESENTATIONS ON LOCALLY CONVEX SPACES}

A. Notation, terminology and basic facts. By a locally convex space we shall in this paper mean a locally convex, Hausdorff, topological vector space over the field of the complex numbers $\mathbb{C}$.

Let $E$ be a locally convex space. Its strong topological dual will be denoted by $E^{\prime}$. Furthermore $L(E)$, resp. $S(E)$, resp. $B(E)$, denotes the vector space of those linear maps of $E$ into $E$, which are continuous, resp. are continuous with respect to the weak topology $\sigma\left(E, E^{\prime}\right)$, resp. map the bounded subsets of $E$ into bounded subsets.

The ultraweak topology is the weakest locally convex topology on $B(E)$ for which the linear functionals

$$
T \rightarrow \sum_{j=1}^{\infty} \lambda_{j}\left\langle T e_{j}, e_{j}^{\prime}\right\rangle
$$

on $B(E)$ are continuous. Here $\left\{\lambda_{j}\right\}$ ranges over $l^{1}(\mathbb{N})$, and $\left\{e_{j}\right\}$ and $\left\{e_{j}^{\prime}\right\}$ range over the bounded sequences in $E$ and $E^{\prime}$ respectively. A subset $A$ of $B(E)$, equipped with the ultraweak topology, will be denoted $A_{\mathrm{uw}}$. 
A representation $\pi$ of a group $G$ on a locally convex space $E$ is (here) a homomorphism of $G$ into the group of invertible elements of $L(E) . \pi$ is said to be ultra-irreducible if $\operatorname{span}\{\pi(G)\}$ is dense in $S(E)_{\mathrm{uw}}$. If so, then $\pi$ is also topologically completely irreducible and hence topologically irreducible, too; also the commutant algebra $\pi(G)^{\prime}$ reduces to the scalars.

A sufficient condition for ultra-irreducibility can be found as Corollary 8 of [LL1] or in [LL2]. It is an infinite dimensional version of Burnside's theorem.

Theorem 1 (Litvinov-Lomonosov). Let $E$ be a locally convex space with the property that the Fredholm operators on it possess a well-defined trace. Then a topologically irreducible representation $\pi$ of a group $G$ on $E$ is ultra-irreducible if the closure of $\operatorname{span}\{\pi(G)\}$ in $L(E)_{\mathrm{uw}}$ contains a nonzero compact operator.

If $\pi$ is a strongly continuous representation of a Lie group $K$ with right Haar measure $d k$ on a locally convex space $E$, then we put

$$
\pi(\phi) u:=\int_{K} \phi(k) \pi(k) u d k \text { for } \phi \in C_{0}^{\infty}(K), u \in E,
$$

where the integral is defined weakly as a linear functional on $E^{\prime}$.

Proposition 2. Let $\pi$ be a strongly continuous representation of a Lie group $K$ on a semicomplete, locally convex space $E$. Then

$(\alpha) \pi(\phi) \in B(E)$ for all $\phi \in C_{0}^{\infty}(K)$.

( $\beta$ ) A closed subspace of $E$ is invariant under $\pi(K)$ iff it is invariant under $\pi\left(C_{0}^{\infty}(K)\right)$.

( $\gamma) \operatorname{span}\left\{\pi(\phi) e \mid \phi \in C_{0}^{\infty}(K), e \in E\right\}$ is dense in $E$.

$(\delta) \operatorname{span}\{\pi(K)\}$ and $\pi\left(C_{0}^{\infty}(K)\right)$ have identical closures in $B(E)_{\mathrm{uw}}$.

B. The abstract set-up and consequences. Throughout this subsection we enforce the conditions of the Abstract Set-Up below.

The Abstract Set-Up 3. $C$ and $E$ are semicomplete, locally convex spaces. $C$ is a subspace of $E$ and the inclusion map $i: C \subset E$ is continuous. $G$ is a Lie group, $K$ is a Lie subgroup of $G$ and $d k$ denotes a right Haar measure on $K$.

$\pi_{C}$ is a strongly continuous representation of $G$ on $C$ that extends to a strongly continuous representation $\pi$ of $G$ on $E$ in such a way that the following holds:

$\pi(\phi) E \subset C$ for each $\phi \in C_{0}^{\infty}(K)$ and the corresponding map $\pi_{E}^{C}(\phi)$ of $E$ into $C$ is continuous.

Easy consequences are that $\pi(\phi) \in L(E)$ and $\pi_{C}(\phi) \in L(C)$ for any $\phi \in$ $C_{0}^{\infty}(K)$, and the following two lemmas:

Lemma 4 (The approximation lemma). If $W$ is a closed, invariant subspace of $E$, then $W \cap C$ is dense in $W$.

Lemma 5. Let $W$ be an invariant subspace of $C$. Then $W$ is dense in $C$ iff it is dense in $E$. 
Theorem 6. $\pi$ is ultra-irreducible iff $\pi_{C}$ is also.

Proof. We shall only give the if part, because the other one can be treated quite similarly. So assume $\pi_{C}$ is ultra-irreducible. To prove $\pi$ is ultra-irreducible it suffices by the Hahn-Banach theorem to check the following implication for given $u \in\left[B(E)_{\mathrm{uw}}\right]^{\prime}$ :

$$
\langle u, \pi(g)\rangle=0 \text { for all } g \in G
$$

implies

$$
\langle u, T\rangle=0 \text { for all } T \in S(E) .
$$

Let (1) be given. We then get

$$
\langle u, \pi(g) \pi(k)\rangle=0 \text { for all } g \in G \text { and } k \in K \text {. }
$$

Composition by an element from $S(E)$, in particular by $\pi(g)$, is continuous with respect to the ultraweak topology, so from Proposition $2(\delta)$ we get that

$$
\langle u, \pi(g) \pi(\phi)\rangle=0 \text { for all } g \in G \text { and } \phi \in C_{0}^{\infty}(K),
$$

which tells us that the linear functional $T_{C} \rightarrow\left\langle u, i \circ T_{C} \circ \pi_{E}^{C}(\phi)\right\rangle$ on $B(C)$ vanishes on $\pi_{C}(G) \subset L(C)$. By the ultra-irreducibility of $\pi_{C}$ it vanishes on all of $S(C)$, so

$$
\left\langle u, i \circ T_{C} \circ \pi_{E}^{C}(\phi)\right\rangle=0 \text { for all } T_{C} \in S(C) .
$$

Given $T \in S(E)$ we replace $T_{C}$ in (3) by $\pi_{E}^{C}\left(\phi_{1}\right) \circ T \circ i \in S(C)$, where $\phi_{1} \in C_{0}^{\infty}(K)$ is arbitrary, to get

$$
\begin{aligned}
0 & =\left\langle u, i \circ \pi_{E}^{C}\left(\phi_{1}\right) \circ T \circ \pi_{E}^{C}(\phi)\right\rangle \\
& =\left\langle u, \pi\left(\phi_{1}\right) \circ T \circ \pi(\phi)\right\rangle .
\end{aligned}
$$

The continuity of left and right compositions by elements from $L(E)$ combined with Proposition $2(\delta)$ now ensures that $(2)$ holds.

\section{THE REPRESENTATIONS $I^{\Delta}$ AND $I^{\Delta \mu}$}

Throughout this section $G$ denotes a second countable Lie group which is the semidirect product $G=S \times{ }_{s} K$ of a normal, closed, and connected subgroup $S$ with a closed subgroup $K$. We let $V$ be a finite-dimensional complex vector space.

We give $C^{\infty}(K, V)$ and $C_{0}^{\infty}(K, V)=\mathscr{D}(K, V)$ their usual topologies (Fréchet space and $L F$-space respectively) and put $\mathscr{D}^{\prime}(K, V):=\left[\mathscr{D}\left(K, V^{\prime}\right)\right]^{\prime}$. We identify $C^{\infty}(K, V)$ as a dense subspace of $\mathscr{D}^{\prime}(K, V)$ via the natural continuous injection map $i: C^{\infty}(K, V) \subset \mathscr{D}^{\prime}(K, V)$ given by

$$
\langle\text { if }, \psi\rangle:=\int_{K}\langle f(k), \psi(k)\rangle d k \text { for } f \in C^{\infty}(K, V), \psi \in C_{0}^{\infty}\left(K, V^{\prime}\right) .
$$

We note that $C^{\infty}(K, V)$ and $\mathscr{D}^{\prime}(K, V)$ are $C^{\infty}(K)$-modules. 
Let $\Lambda$ be a continuous homomorphism from $S$ to $\mathbb{C} \backslash\{0\}$. By Lie group theory, $\Lambda \in C^{\infty}(S)$. We define a continuous representation $I^{\Lambda}$ of $G$ on $C^{\infty}(K, V)$ by (cf. [Wi, formula (2.8), p. 72] )

$$
\left[I^{\Lambda}(s k) f\right]\left(k_{1}\right):=\Lambda\left(k_{1} s k_{1}^{-1}\right) f\left(k_{1} k\right)
$$

for $\left(s, k, k_{1}\right) \in S \times K \times K, f \in C^{\infty}(K, V)$.

Definition 1. For $u \in \mathscr{D}^{\prime}(K, V), \phi \in C_{0}^{\infty}\left(K, V^{\prime}\right)$ and $(s, k) \in S \times K$ we put

$$
\left\langle I^{\Lambda}(s k) u, \phi\right\rangle:=\left\langle u, I^{\Lambda}\left(k^{-1} s\right) \phi\right\rangle .
$$

$I^{\Lambda}$, defined by (2), is a continuous representation of $G$ on $\mathscr{D}^{\prime}(K, V)$, extending the representation (1) on $C^{\infty}(K, V)$. The stability subgroup

$$
M=K_{\Lambda}:=\left\{k \in K \mid \Lambda\left(k s k^{-1}\right)=\Lambda(s) \text { for all } s \in S\right\}
$$

is a closed subgroup of $K$. Let $\mu$ be a continuous, topologically irreducible representation of $M$ on a complex finite dimensional vector space $V=H(\mu)$. $M$ acts on $C^{\infty}(K, H(\mu))$ (or more generally on functions from $K$ to $H(\mu)$ ) by

(3) $[m \cdot \phi](k):=\mu(m)\left[\phi\left(m^{-1} k\right)\right]$ for $m \in M, k \in K, \phi \in C^{\infty}(K, H(\mu))$.

$\phi$ is a fixed point iff $\phi$ satisfies the covariance condition $(*)$ from the Introduction.

The action of $M$ extends to a continuous representation of $M$ on $\mathscr{D}^{\prime}(K, H(\mu))$ which commutes with $I^{\Lambda}$ and multiplication by functions from $C^{\infty}(M \backslash K)$. The vector space

$$
\begin{aligned}
\mathscr{D}_{\mu}^{\prime} & :=\left\{u \in \mathscr{D}^{\prime}(K, H(\mu)) \mid m \cdot u=u \text { for all } m \in M\right\}, \\
C_{\mu}^{\infty} & :=C^{\infty}(K, H(\mu)) \cap \mathscr{D}_{\mu}^{\prime} \text { and } C_{\mu}:=C(K, H(\mu)) \cap C_{\mu}^{\infty}
\end{aligned}
$$

are therefore invariant under $I^{\Lambda}$ and multiplication by functions from $C^{\infty}(M \backslash K)$. They are closed subspaces of $\mathscr{D}^{\prime}(K, H(\mu)), C^{\infty}(K, H(\mu))$ and $C(K, H(\mu))$ respectively. We equip them with the topologies from

$$
\mathscr{D}^{\prime}(K, H(\mu)), C^{\infty}(K, H(\mu))
$$

and $C(K, H(\mu))$ respectively. The restriction $I^{\Lambda \mu}$ of $I^{\Lambda}$ from $\mathscr{D}^{\prime}(K, H(\mu))$ to $\mathscr{D}_{\mu}^{\prime}$ is then a continuous representation of $G$ on $\mathscr{D}_{\mu}^{\prime}$; restricting further we get that $I^{\Lambda \mu}$ defines a continuous representation (again denoted $I^{\Lambda \mu}$ ) of $G$ on $C_{\mu}^{\infty}$.

Remark 2. The Abstract Set-Up holds here with $C=C_{\mu}^{\infty}, E=\mathscr{D}_{\mu}^{\prime}$ and $\pi=$ $I^{\Lambda \mu}$.

There is a continuous projection $p$ of $C^{\infty}(K, H(\mu))$ onto $C_{\mu}^{\infty}$. It can be constructed as follows: Choose $\theta \in C^{\infty}(K)$ such that $\operatorname{supp}\{m \rightarrow \theta(m k) \mid k \in$ $Q$ \} is compact for any compact subset $Q$ of $K$ and such that

$$
\int_{M} \theta(m k) d m=1 \quad \text { for each } k \in K
$$


(cf. the proof of Lemma A.1.1 of [Wa] for the existence of such a $\theta$ ).

Then

$$
(P k)(k):=\int_{M} \theta(m k) \mu(m)^{-1}[f(m k)] d m,
$$

where $d m$ is a right Haar measure on $M$, works.

Our final result of this section is the first place in which the irreducibility of $\mu$ is used. So far $\operatorname{dim} H(\mu)<\infty$ has sufficed (cf. [Wi, Lemma 3.1; Th, Lemma 3]).

Lemma 3. If $\mathscr{A}$ is a dense subset of $C^{\infty}(M \backslash K)$ and $w \in C_{\mu}^{\infty} \backslash\{0\}$, then $\operatorname{span}\left\{a I^{\Lambda}(k) w \mid a \in \mathscr{A}, k \in K\right\}$ is dense in $C_{\mu}^{\infty}$.

Proof. Since the map $a \rightarrow a f$ for any fixed $f \in C_{\mu}^{\infty}$ is continuous from $C^{\infty}(M \backslash K)$ into $C_{\mu}^{\infty}$ we get that the closure of $\operatorname{span}\left\{a I^{\Lambda}(k) w \mid a \in \mathscr{A}, k \in K\right\}$ contains $\operatorname{span}\left\{\phi I^{\Lambda}(k) w \mid \phi \in C^{\infty}(M \backslash K), k \in K\right\}$, so that we may assume that $\mathscr{A}=C^{\infty}(M \backslash K)$. But in that case $\operatorname{span}\left\{a I^{\Lambda}(k) w \mid a \in \mathscr{A}, k \in K\right\}$ contains $P\left(\operatorname{span}\left\{\phi I^{\Lambda}(k) w \mid \phi \in C^{\infty}(K), k \in K\right\}\right)$. Since $P$ is surjective it suffices to prove that $\operatorname{span}\left\{\phi I^{\Lambda}(k) w \mid \phi \in C^{\infty}(K), k \in K\right\}$ is dense in $C^{\infty}(K, V)$.

Replacing $w$ by a suitable translate we may assume that $w(e) \neq 0$.

We shall show that the annihilator in $C^{\infty}(K, V)^{\prime}$ of

$$
\operatorname{span}\left\{\phi I^{\Lambda}(k) w \mid \phi \in C^{\infty}(K), k \in K\right\}
$$

is $\{0\}$.

By Lemma II.4 it suffices to prove that if $U \in C_{0}^{\infty}\left(K, V^{\prime}\right)$ is in the annihilator then $U=0$. So assume that $U$ is in the annihilator. Then for all $a \in C^{\infty}(K)$ and $k_{0} \in K$ we have

$$
\begin{aligned}
0 & =\left\langle U, a I^{\Lambda}\left(k_{0}\right) w\right\rangle=\int_{K}\left\langle U(k), a(k)\left(I^{\Lambda}\left(k_{0}\right) w\right)(k)\right\rangle d k \\
& =\int_{K} a(k)\left\langle U(k),\left(I^{\Lambda}\left(k_{0}\right) w\right)(k)\right\rangle d k,
\end{aligned}
$$

so

$$
0=\left\langle U(k),\left(I^{\Lambda}\left(k_{0}\right) w\right)(k)\right\rangle=\left\langle U(k), w\left(k k_{0}\right)\right\rangle \text { for all } k, k_{0} \in K .
$$

Choosing $k_{0}$ suitably we get for any $m \in M$ that

$$
0=\langle U(k), w(m)\rangle=\langle U(k), \mu(m)[w(e)]\rangle .
$$

Since $w(e) \neq 0$ and $\mu$ is irreducible we get that $U(k)=0$. But $k \in K$ was arbitrary.

\section{The POISSON TRANSFORM}

The situation and notation are as in $\S$ III.

The Lie group $G$ is the semidirect product of a normal, closed and connected subgroup $S$ with a closed subgroup $K$. k will denote the Lie algebra of $K$ 
and $L(S)$ that of $S . \Lambda$ is a smooth homomorphism of $S$ into $\mathbb{C} \backslash\{0\}$, and $M$ denotes the stability subgroup $M=K_{\Lambda}$.

We assume that there exists an $\operatorname{Ad}(K)$-invariant inner product $\langle\cdot, \cdot\rangle$ on $L(S)$, and extend it to $L(S)^{\mathbb{C}}$. Define $d \Lambda \in L(S)^{\mathbb{C}}$ by (cf. [Wi, p. 70])

$$
\Lambda(\exp X)=e^{\langle X, d \Lambda\rangle} \quad \text { for } X \in L(S),
$$

define the functions $\phi_{d \Lambda, Z} \in C^{\infty}(M \backslash K)$ for $Z \in L(S)^{\mathbb{C}}$ by

$$
\phi_{d \Lambda, Z}:=e^{\langle\operatorname{Ad}(k) Z, d \Lambda\rangle} \quad \text { for } k \in K,
$$

and define the $K$-invariant, point separating subalgebras

$$
\mathscr{A}_{0}(d \Lambda):=\operatorname{span}\left\{\phi_{d \Lambda, Z} \mid Z \in L(S)\right\}, \quad \mathscr{A}(d \Lambda):=\operatorname{span}\left\{\phi_{d \Lambda, Z} \mid Z \in L(S)^{\mathbb{C}}\right\}
$$

of $C^{\infty}(M \backslash K)$ [Wi, p. 74 and Proposition 4.4]. Note that $\mathscr{A}_{0}(d \Lambda)$ and $\mathscr{A}(d \Lambda)$ have the same closures in $C^{\infty}(M \backslash K)$ (cf. the proof of Proposition 1 below).

We will for brevity write $k \cdot Z$ for $\operatorname{Ad}(k) Z$, and $X \cdot Z$ for the action of $X \in \mathfrak{k}$ on $Z \in L(S)^{\mathbb{C}}$.

Let $\sigma$ be a smooth volume element on $M \backslash K$ and let $\rho \in C^{\infty}(M \backslash K \times K)$ be the function satisfying

$$
\int_{M \backslash K} \psi(x k) d \sigma(x)=\int_{M \backslash K} \psi(x) \rho(x, k) d \sigma(x) \quad \text { for all } \psi \in C_{0}^{\infty}(M \backslash K) \text {. }
$$

We define the Poisson transformation $P=P_{d \Lambda}: C_{0}^{\infty}(M \backslash K) \rightarrow C^{\infty}(L(S))$ by

$$
(P f)(X):=\int_{M \backslash K} \phi_{d \Lambda, X}(x) f(x) d \sigma(x) \text { for } X \in L(S) .
$$

Proposition 1. $P$ is injective iff $\mathscr{A}(d \Lambda)$ is dense in $C^{\infty}(M \backslash K)$. This is in particular the case if $\mathscr{A}(d \Lambda)$ is closed under complex conjugation.

Proof. Assume first that $\mathscr{A}(d \Lambda)$ is dense in $C^{\infty}(M \backslash K)$ and that

$$
\int_{M \backslash K} \phi_{d \Lambda, X}(x) f(x) d \sigma(x)=0 \text { for some } f \in C_{0}^{\infty}(M \backslash K) \text {. }
$$

Since the map $Z \rightarrow \int_{M \backslash K} \phi_{d \Lambda, Z}(x) f(x) d \sigma(x)$ is holomorphic on $L(S)^{\mathbb{C}}$ and vanishes on the real part $L(S)$ of $L(S)^{\mathbb{C}}$, it vanishes everywhere. So

$$
\int_{M \backslash K} \phi_{d \Lambda, Z}(x) f(x) d \sigma(x)=0 \text { for all } Z \in L(S)^{\mathbb{C}} \text {. }
$$

Since $\mathscr{A}(d \Lambda)$ is dense in $C^{\infty}(M \backslash K), f$ has to be 0 .

Assume conversely that $P$ is injective. By the Hahn-Banach theorem we shall show the following: If $f \in \mathscr{E}^{\prime}(M \backslash K)$ annihilates $\mathscr{A}(d \Lambda)$ then $f=0$.

By the Approximation Lemma II.4 we may assume that $f \in C_{0}^{\infty}(M \backslash K)$. So we know that

$$
\int_{M \backslash K} \phi_{d \Lambda, Z}(x) f(x) d \sigma(x)=0 \text { for all } Z \in L(S)^{\mathbb{C}} .
$$


By the injectivity of $P$ we see that $f=0$ as desired.

The last statement follows from Stone-Weierstrass' theorem and Lemma II.5.

The papers [Th, Wi and Ra] contain various sufficient conditions for $\mathscr{A}(d \Lambda)$ to be dense in $C^{\infty}(M \backslash K)$ when $K$ is compact. (Note that $\mathscr{A}(d \Lambda)$ is dense in $C^{\infty}(M \backslash K)$ iff it is dense in $L^{2}(M \backslash K)$ (Lemma II.5).)

[He, CD and Ko] show that the Poisson transform is injective when $G$ is the Cartan motion group. We present an extension to semidirect products, using an idea due to Koranyi [Ko]:

Theorem 2. The Poisson transform is injective if

$$
\langle X \cdot d \Lambda, d \Lambda\rangle=0 \text { for all } X \in \mathfrak{k} .
$$

Remarks. (i) Condition (2) is automatically satisfied in the case of the Cartan motion group. (Combine (a) and (b), p. 295, of [Ko]. It hinges on the fact that $\operatorname{Re} \Lambda$ and $\operatorname{Im} \Lambda$ commute, both being in the (maximal) commutative subalgebra a.)

(ii) [Wi, Theorem 5.3, p. 78] proves that $\mathscr{A}(d \Lambda)$ is dense in $C(M \backslash K)$ if the function $k \rightarrow\langle k \cdot d \Lambda, d \Lambda\rangle$ is real-valued and $K$ is compact. A particular case occurs if $d \Lambda$ is proportional to a vector in $L(S)$ [Th, Theorem 4]. Our condition (2) is weaker.

(iii) Another connection is to vectors of minimal length (cf. [KN]): If $d \Lambda$ is of minimal length with respect to $K^{\mathbb{C}}$ then (2) holds, (see also [Ra, §2.4]).

Proof of Theorem. Let $m:=\frac{1}{2} \operatorname{dim} M \backslash K$ and $o:=M 1 \in M \backslash K$. We shall apply the method of stationary phase for complex-valued phase functions (Theorem 2.3 of [MS] or formula $(\mathrm{X} .3 .5)$, p. 536, of [Tr] $)$ to prove $\exp (-t\langle d \Lambda, d \Lambda\rangle)$ times the integral

$$
P_{d \Lambda}(f)(t d \Lambda)=\int_{M \backslash K} e^{t\langle x \cdot d \Lambda, d \Lambda\rangle} f(x) d \sigma(x) \text { as } t \rightarrow \infty,
$$

where $f \in C_{0}^{\infty}(M \backslash K)$, behaves asymptotically as $c t^{-m} f(o)$, where $c$ is a nonzero constant that does not depend on $f$. To that purpose we introduce the function $a \in C^{\infty}(M \backslash K)$ defined by

$$
a(x):=i\{\langle d \Lambda, d \Lambda\rangle-\langle x \cdot d \Lambda, d \Lambda\rangle\} \text { for } x \in M \backslash K,
$$

and shall then prove that

$$
\int e^{i t a(x)} f(x) d \sigma(x) \approx c t^{-m} f(o)+\cdots \quad \text { as } t \rightarrow \infty .
$$

Since $K$ acts by orthogonal transformations we see that

$$
\operatorname{Im} a \geq 0 \text { with equality only at } x=0,
$$

because $M=\{k \in K \mid k \cdot d \Lambda=d \Lambda\}$ [Wi, Proposition 4.3, p. 74].

For any $X \in \mathfrak{k}$ we have

$$
X a=-\left.i \frac{d}{d t}\right|_{t=0}\langle\exp (t X) \cdot d \Lambda, d \Lambda\rangle=-i\langle X \cdot d \Lambda, d \Lambda\rangle=0
$$


by our assumption (2), so $x=o$ is a critical point of $a$. For any $X \in \mathfrak{k}$ we compute:

$$
\begin{aligned}
\left.\frac{d^{2}}{d t^{2}}\right|_{t=0} a(M \exp t X) & =\left.(-i) \frac{d^{2}}{d t^{2}}\right|_{t=0}\langle\exp (t X) \cdot d \Lambda, d \Lambda\rangle \\
& =(-i)\left\langle X^{2} \cdot d \Lambda, d \Lambda\right\rangle=i|| X \cdot d \Lambda \|^{2} .
\end{aligned}
$$

This is different from zero for any $X \in \mathfrak{k}$ off the Lie algebra of $M$, so $x=o$ is nondegenerate.

Theorem 2.3 of [MS] applies to functions supported in a sufficiently small neighborhood $U$ of the critical point in question, so let us write $f=F+$ $f_{o}$, where $f_{o}=f$ near $o$ and $\operatorname{supp} f_{o} \subset U$. It is enough to show that $F$ contributes nothing to the first term of the formula for the asymptotic behaviour.

By (3) there exists a $\delta>0$ such that $\operatorname{Im} a \geq \delta$ on $\operatorname{supp} F$, so

$$
\left|\int e^{i t a(x)} F(x) d \sigma(x)\right| \leq \int e^{-t \operatorname{Im} a(x)}|F(x)| d \sigma(x) \leq e^{-t \delta} \int|F(x)| d \sigma(x),
$$

so $F$ does indeed contribute nothing to the asymptotic expansion.

At last we turn to the Poisson transform. We shall show that

$$
\left\{f \in C_{0}^{\infty}(M \backslash K) \mid P_{d \Lambda} f(X)=0 \text { for all } X \in L(S)\right\}=\{0\} .
$$

The asymptotics show us that $f(o)=0$ for any $f$ in the left-hand side. If $f$ belongs to the left-hand side then so does $I^{\Lambda}(k)\left[f \rho(\cdot, k)^{-1}\right]$ for any $k \in K$, so

$$
0=I^{\Lambda}(k)\left[f \rho(\cdot, k)^{-1}\right](o)=f(M k) \rho(M k, k)^{-1}
$$

and hence $f(M k)=0$.

\section{A SUFFICIENT CONDITION FOR ULTRA-IRRREDUCIBILITY}

The situation is as stated in $\S \mathrm{IV}$. Furthermore $\mu$ is a continuous, irreducible and finite dimensional representation of $M$ on $H(\mu)=V$.

Proposition 1. The representation $I^{\Lambda \mu}$ of $G$ is ultra-irreducible on $C_{\mu}^{\infty}$ if $\mathscr{A}_{0}(d \Lambda)$ is dense in $C^{\infty}(M \backslash K)$.

Proof. Let $W \neq\{0\}$ be an invariant subspace of $C_{\mu}^{\infty}$. W is in particular invariant under $I^{\Lambda \mu}(\exp X)=\phi_{d \Lambda, X} \in \mathscr{A}_{0}(d \Lambda)$, so by Lemma III.3 $W$ is dense in $C_{\mu}^{\infty}$. This proves that $I^{\Lambda \mu}$ is topologically irreducible. We continue by applying the Litvinov-Lomonosov result (Theorem II.1):

$C_{\mu}^{\infty}$ has that property that the Fredholm operators on it have well-defined traces, because there exists a continuous projection $P$ of $C^{\infty}(K, V)$ onto it, and $C^{\infty}(K, V)$ has the property (see e.g. [JS]).

Via Proposition II.2 $(\delta)$ we see that $a I^{\Lambda \mu}(\phi) \in \operatorname{span}\{\pi(G)\}_{\text {uw }}$ for any $a \in$ $\mathscr{A}_{0}(d \Lambda)$ and $\phi \in C_{0}^{\infty}(K)$, and hence by density even for all $a \in C^{\infty}(M \backslash K)$, so it suffices to prove that $a I^{\Lambda \mu}(\phi)$ is compact when $a \in C_{0}^{\infty}(M \backslash K)$. Now 
$a=P(\psi)$ for some $\psi \in C_{0}^{\infty}(K)$, so for any $f \in C_{\mu}^{\infty}$ :

$$
a I^{\Lambda \mu}(\phi) f=P(\psi) I^{\Lambda \mu}(\phi) f=P\left(\psi I^{\Lambda}(\phi) f\right) .
$$

It now suffices to show that $\psi I^{\Lambda}(\phi): C^{\infty}(K, V) \rightarrow C^{\infty}(K, V)$ is compact. But here we may assume $V=\mathbb{C}$ because there is no twisting any longer. $\psi I^{\Lambda}(\phi)$ is compact as an integral operator with smooth, compactly supported kernel.

The next theorem, which is our main result, deals with spaces of functions and distributions between $C_{\mu}^{\infty}$ and $\mathscr{D}_{\mu}^{\prime}$ that satisfy the covariance condition (*), e.g. $C_{\mu}(K, H(\mu))$ and, for $K$ compact, $L_{\mu}^{p}(K, H(\mu))$ for $1 \leq p<\infty$.

Theorem 4.11 of [Wi] and Theorem 4 of [Th] are special cases.

Theorem 2. Let $F$ be a semicomplete, locally convex space which is a subspace of $\mathscr{D}_{\mu}^{\prime}$ such that $C_{\mu}^{\infty} \subset F \subset \mathscr{D}_{\mu}^{\prime}$ with continuous inclusions.

Assume that $F$ is $I^{\Lambda \mu}$-invariant, and that the map $g \rightarrow I^{\Lambda \mu}(g) \mid F=I_{F}(g)$ is a strongly continuous representation of $G$ on $F$.

Then $I_{F}$ is an ultra-irreducible representation, if the Poisson transform is injective.

Proof. The theorem is an immediate consequence of Proposition 1 in view of Theorem II.6.

Remark 3. Theorem 1 provides us with a proof of the "double commutant" theorem in [Wi, Theorem 1.3]: Indeed, if $\mathscr{A}(d \Lambda)$ is closed under complex conjugation then it is dense in $C^{\infty}(M \backslash K)$, so $I^{\Lambda \mu}$ is ultra-irreducible (Theorem $1)$ and thus topologically completely irreducible.

Example 4. In case of the Euclidean motion group $\left(S=\mathbb{R}^{n}, K=S O(n)\right)$ our condition (2) holds iff $d \Lambda$ is proportional to a real vector. It is known that for $n>2$ this is necessary and sufficient for $I^{\Lambda \tau}$ to be (topologically) irreducible, $\tau$ being the trivial representation [Ra, 3.5, Exemples]. According to our theorem we even get ultra-irreducibility in that case.

\section{ApPlication to the CARTAN MOtion GROUP}

Standard notation. Let $G$ be a semisimple, connected, real Lie group with finite center, and let $\mathfrak{g}$ be its Lie algebra. Let $\mathfrak{g}=\mathfrak{k}+\mathfrak{p}$ be a Cartan decomposition of $\mathfrak{g}$ and $K$ be the corresponding maximal compact subgroup. $G_{0}:=\mathfrak{p} \times{ }_{s} K$ is the Cartan motion group of (some) affine motions of $\mathfrak{p}$.

We let $\mathfrak{a}$ be a maximal abelian subspace of $\mathfrak{p}$, let $\lambda: \mathfrak{a} \rightarrow \mathbb{C}$ be real-linear, and let $K^{\lambda}$ denote the stabilizer of $\lambda$ in $K$.

As is usual we extend $\lambda$ to all of $\mathfrak{p}$ by setting it 0 on the orthogonal complement of $\mathfrak{a}$ in $\mathfrak{p}$ with respect to the Killing form.

We define the continuous homomorphism $\Lambda: \mathfrak{p} \rightarrow \mathbb{C} \backslash\{0\}$ by

$$
\Lambda(X):=e^{i \Lambda(X)} \quad \text { for } X \in \mathfrak{p},
$$

and note that $K_{\Lambda}=K^{\lambda}$. 
Let $\mu$ be a continuous, irreducible representation of $K^{\lambda}$ on $H(\mu)$. From formula (III.1) above we get a representation $I^{\lambda}$ of $G_{0}$ on $C^{\infty}(K, H(\mu))$ given by

$$
\begin{aligned}
& {\left[I^{\lambda}(X, k) f\right]\left(k^{\prime}\right)=e^{i \lambda\left(\operatorname{Ad}\left(k^{\prime}\right) X\right)} f\left(k^{\prime} K\right)} \\
& \quad \text { for } X \in \mathfrak{p}, k, k^{\prime} \in K \text { and } f \in C^{\infty}(K, H(\mu)) .
\end{aligned}
$$

The representation extends to $\mathscr{D}^{\prime}(K, H(\mu))$ as before.

By the injectiveness of the Poisson transform on the Cartan motion group we get from the previous results:

Theorem 1. Let $F$ be a semicomplete, locally convex space such that $C_{\mu}^{\infty} \subset$ $F \subset \mathscr{D}_{\mu}^{\prime}$ with continuous inclusions.

If $F$ is $I^{\lambda}$-invariant and the restriction $I_{F}$ of $I^{\lambda}$ to $F$ is a strongly continuous representation of $G_{0}$ on $F$, then $I_{F}$ is ultra-irreducible.

The theorem holds in particular for the representation $I^{\lambda}$ on the space $F=$ $L_{\mu}^{2}(K, H(\mu))$, which is the one considered in [CD, Théorème 6, p. 278]. By other methods than ours, [CD] established that $I^{\lambda}$ is topologically completely irreducible.

\section{REFERENCES}

[CD] C. Champetier and P. Delorme, Sur les représentations des groupes de déplacements de Cartan, J. Funct. Anal. 43 (1981), 258-279. MR 83e: 22012

[Gi] S. G. Gindikin, Unitary representations of groups of automorphisms of Riemann symmetric spaces of null curvature, Functional Anal. Appl. 1 (1967), 28-32. MR 35\# 303

[He] S. Helgason, $A$ duality for symmetric spaces with applications to group representations. III. Tangent space analysis, Adv. in Math. 30 (1980), 297-323. MR 81g : 22021.

[JS] Jacob Jacobsen and Henrik Stetkær, Spaces in which Fredholm operators have well defined trace, J. Operator Theory 19 (1988), 381-386.

[KN] G. Kempf and L. Ness, The length of vectors in representation spaces, in Algebraic Geometry, Proceedings Copenhagen 1978 (K. Lonsted, ed.), Lecture Notes in Math., vol. no 732, Springer-Verlag, Berlin, Heidelberg and New York, 1978, pp. 233-243. MR 81i : 14032.

[Ko] A. Koranyi, On the injectivity of the Poisson transform, J. Funct. Anal. 45 (1982), 293-296. MR 84i : 43008.

[LL1] G. L. Litvinov and V. I. Lomonosov, Density theorems in locally convex spaces and their applications, Trudy Sem. Vektor. Tenzor Anal. 20 (1981), 210-227. (Russian) MR 83i \#47009.

[LL2] _ Density theorems in locally convex spaces and irreducible representations, Soviet Math. Dokl. 23 (1981), 372-376; English transl., Dokl. Akad. Nauk SSSR 257 (1981), no. 4, 826830. MR 82g \#46087.

[MS] A. Melin and J. Sjöstrand, Fourier integral operators with complex-valued phase functions, in Fourier Integral Operators and Partial Differential Equations, Colloq. Internat., Nice 1974 (J. Chazarain, ed.), Lecture Notes in Math., vol. 459, Springer-Verlag, Berlin and New York, 1975, pp. 120-233. MR 55 \#4290.

[Ra] M. Raïs, Sur l'irréductibilité de certaines représentations induites non unitaires, C. R. Acad. Sci. Paris, Sér. I 305 (1987), 713-716.

[Tr] F. Treves, Introduction to pseudodifferential and Fourier integral operators, vol. 2, Plenum Press, New York and London, 1980. 
[Th] E. Thieleker, On the irreducibility of nonunitary induced representations of certain semidirect products, Trans. Amer. Math. Soc. 164 (1972), 353-369. MR 45 \#2097

[Wa] G. Warner, Harmonic analysis on semi-simple Lie groups. I, Springer-Verlag, Berlin, Heidelberg and New York, 1972.

[Wi] F. L. Williams, Topological irreducibility of nonunitary representations of group extensions, Trans. Amer. Math. Soc. 233 (1977), 69-84. MR 57 \#3316

Department of Mathematics, Aarhus University, Ny Munkegade, DK-8000 Aarhus C, DENMARK 